\title{
The Research and Design of Intelligent tutoring System in Intelligent Turtoring System based on Campus Grid
}

\author{
Xia Yaowen and Xie Jili \\ Institute of Information \\ Yunnan Normal University \\ Kunming,Yunnan Province,China \\ xywen_km@sina.com
}

\author{
Lv Saidong and Tang Guohua \\ Institute of Security \\ Yunnan Normal University \\ Kunming,Yunnan Province,China \\ lsdong_km@sina.com
}

\begin{abstract}
Grid technology has become the computer and network technology's research hotspot and frontier, Grid technology development and the consummation will promote the development of educational information. The intelligent has shortcoming which are resource isolated, update difficulty etc, Based on the grid technology research and application, using OGSA (Open Grid Services Architecture) grid system structure, Taking GLOBUS as the grid middleware to build grid platform. This paper discuss the educational grid minimum implementation unit - - teaching system of campus grid and put forward the network teaching system model based on the grid technology. Then establish a distributed, heterogeneous sharing information resources of the library, it provides an efficient, fully shared virtual teaching space for network tutoring system. Finally, intelligent network teaching system and the grid binding laid certain foundation.
\end{abstract}

Keywords-grid, heterogeneous, campus grid, grid technology, intelligent turtoring system

\section{INTRODUCTION}

With the rapid development of network technology, network teaching has unique advantages that it's rich teaching resources, friendly interactive. It has become a new, practical teaching mode. The most prominent feature of the network teaching pattern is the use of modern technology, broke through the time and spatial boundaries, realize the teaching resources integration and sharing of maximum range and fill the traditional classroom teaching process in the presence of a blank, then effectively improve the efficiency of teaching and studying. Network intelligent teaching model for teachers and students to provide an independent, personalized, open education software environment, but also for the development of educational information plays a good role in promoting. But the current network teaching system basically is the use of established model, namely a variety of learning tools; lack of learning needs and resources integration, a huge amount of information, the media categories, scattered storage, behave formal diversity and continuous dynamic increase. Existing resource basically is a specific mode for centralized storage, dynamic update and independent access, influence the efficiency of construction.

Generally, network learning need handle a large number of isomorphic or heterogeneous learning information in dynamic distributed environments. In the network learning,
Learners request service is not a single learning resource, but through a variety of resources cooperate to complete.

In addition due to the University District and professional independence, the network teaching system in Colleges and universities are mostly their own software and hardware condition, The teaching system is not completely interconnection, sharing and cooperation. It has become a constraining the development of educational information technology bottleneck, its limitations for specific performance:

- Universities and professional independently developed their own network teaching system, so it has caused the same professional resource repeated construction, unnecessary waste of manpower, financial resources;

- The system lacks a unified management of resource retrieval ability, information messy, dispersion and scheduling. It is difficult for students from the complicated resources in search of their real need information.

- The lack of excellent cyber source, too few resources, between various universities did not achieve the true sense of the sharing of resources, so some excellent resources cannot learn from each other and exchange.

- Computer hardware and software updates system compatibility is not high, dynamic maintenance problems.

- Therefore, how to use existing technology, build powerful function, reasonable structure of the network teaching system, it is the urgent need to consider and solve important problem.

This paper is based on the current network teaching development status and requirements, design and implementation of a network teaching system based on grid technology,

The system realize the sharing of teaching resources and the platform of information resource access [1][2].

\section{ANYSIS OF GRID}

The grid is major progress of science and technology Internet after, it will be geographically distributed heterogeneous range of high-performance computers, data server and large-scale retrieval storage system. Through the network integration connection, unified allocate and use of all resources, establish network service oriented common based on support environment. Realize computing resources 
Internet on and effective polymerization and widely shared of data resources and services resources. Thereby establish a virtual scientific research experimental environment which can realize the regional or global cooperative or collaborative, thus support to large-scale computing and data processing for the characteristics of the scientific activities. The grid can be understood as a system which through a standard, open and universal protocol and interface to coordinate distributed resources in order to provide the best quality of service.

The grid has some basic properties [3]

- Distribution and sharing: A grid system consists of distributed in different locations, different types of computers, peripherals, all kinds of database, knowledge base, grid services composition. Distribution is the most fundamental, the most typical grid and the most important character. The grid is to physically dispersed resources integration, coordination of work, on various resources sharing.

- The dynamic and heterogeneous: Grid is a system, its size, resources, services are in constant change. Grids's dynamic including grid resources, grid size with time changing, or fill in the content, or the removal of unused resources. Heterogeneous refers to the grid in different forms of the computer system, at the same time, information in the grid resources are also diversified, various kinds of media information, different kinds of knowledge database, by different language development of the application software and so on.

- Self similarity: Grid structure with self similar characteristics.

\section{GRID SYSTEM IN COLLEGES AND UNIVERSITIES}

Campus grid is a grid technology implementation in campus. It is intended to be within the campus network resources such as the teaching resources, storage resources, computing resources in an efficient manner dynamic organization. It is intended to be within the campus network resources such as the teaching resources, storage resources, computing resources in an efficient manner dynamic organization, concealed the heterogeneous and dynamic characteristics of grid, to provide users with efficient computing environment and unified transparent grid service. From the application point of view, the future campus network ideal structure as shown in figure 1.

- The first layer (layer) is the campus network resources, mainly by computer, switches, routers and other network hardware resources and databases (or data center resources) etc. The second layer is a grid service implementation, the layer is the realization of campus network service function key,

- The second layer is a grid service implementation; the layer is the realization of campus network service function key.

- $\quad$ The third layer is a grid service interface, through which the external services.
- $\quad$ The fourth layer is applied system which face final user, enjoy the grid service, are in fact through the application system, application system here has played a role of portal.

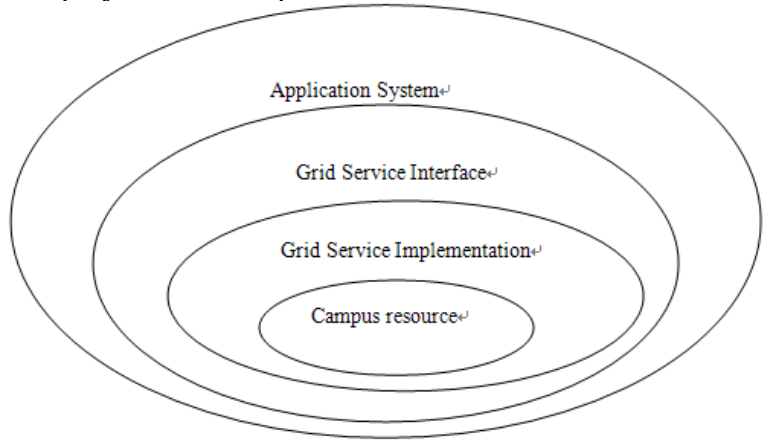

Figure 1. Campus grid structure

\section{GRID ITSDESIGN}

\section{A. Analysis}

Grid is a new network computing platform after World Wide Web. The distribution of the resources integration grid for a huge super computer provides computing resources, storage resources, data resources, information resources, knowledge resources, expert resources, equipment resources sharing [5]. Its purpose is to be in distributed, heterogeneous, autonomous cyber source environment on structural dynamic virtual organizations, and in its internal implementation of cross regional resource sharing and collaboration. The application of grid technology in ITS, ITS can improve various properties, Specific performance in [6]:

- Learning resources are stored in a plurality of different grid nodes; it can realize the transparency of distributed learning resources, management, and use of the resources in the grid without the specified resource location.

- Construction of dynamic virtual learning environment, realize the resource sharing and collaboration between students, to better achieve the cooperative work, to provide a better learning service. For example, students may mutually sharing learning resources, exchange learning experience, mutual feedback information.

- Each grid node is capable of performing local application requests.

\section{B. Intelligent Turtoring System based on Grid}

Remote teaching system of campus grid environment different from the traditional remote teaching system is the key to the server processing, the wide range of resource sharing and service sharing is possible through using grid technology. Therefore, the learners can get more and better services through the campus grid. Combined with the grid heterogeneous shared characteristics, we design follows the grid teaching system model (as shown in Figure 2). In the model, The teaching system (physical resources) are distributed in different geographical locations, 
These resources are distributed, heterogeneous, autonomous, dynamic characteristics. The system merges into a virtual computer, all the physical resource mapping into a virtual resource in grid environment by using of grid technology. The server of these physical resources send to the remote grid virtual teaching system information: Network curriculum, including network course, video streaming course and remote experiment platform, etc. The user through the landing distance grid virtual teaching system for reading information, also can store information for other users, The learner use the virtual resources, transparent operation and sharing, without considering its location and the environment [7][8].

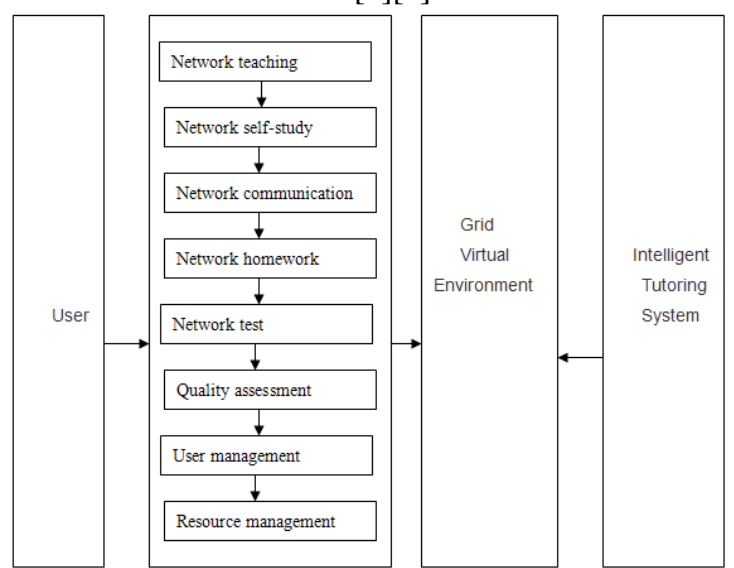

Figure 2. Intelligent tutoring system model base on campus grid

\section{CONCLUSIONS}

Grid technology makes modern educational system resource utilization rate is improved greatly, the user feels great convenience and more experience, It makes the learners' study and work efficiency. The remote education has injected new vitality. However, grid technology is still in its initial stage, each index still need further mature and perfect. The majority of the grid system is still in the stage of theoretical research, the practical application of less. With the grid technology unceasing development and the consummation, it will greatly influence the development of modern distance education. Therefore, using grid computing means to carry out distance education is an inevitable trend; it will bring advantages more and more obvious. This is the research direction in the future.

\section{ACKNOWLEDGMENT}

This work is supported by the National NSF of China (No.60903131), the key project of the Education Bureau Youth Fund of Yunnan Province (No.07Z1066).

\section{REFERENCES}

[1] Mei Hong,Li Jigui.The research of remote distance education system based on grid[J].Compute science,2006,33(6):293-295

[2] Lan Li.Campus network teaching resource sharing of heterogeneous based on grid technology[J].Science and technology information:Academic Edition,2006(5):138-140

[3] Du Zhihui.Grid calculation[M].Beijing:Tsinghua University Press,2002

[4] FOSTER Ian, KESSELMAN Carl. The Grid 2: Blueprint for a New Computing Infrastructure[M]. Jin, Yuan Pingpeng, Shi Ke, transl. Beijing: Publishing House of electronics industry, 2004:230-256.

[5] The Mark, Yin Zhijun, etc.Graves.XML database design [M]. Beijing: Mechanical Industry Press, 2002

[6] Zhong Zhishi. Agent-based Semantic Grid China Agent Systems School[C].Chong qing: South west China Normal University, 2004. $16-22$

[7] Xu Zhiwei, Feng Baiming, Li Wei. Grid computing technology [M]. Beijing: Publishing House of electronics industry, 2005:78-102.

[8] Gui Xiaolin. Grid technology [M]. Beijing: Beijing University of Posts and Telecommunications press, 2005:32-53. 